\title{
Uveitis-Glaucoma-Hyphema Syndrome Following iStent Implantation
}

\author{
Adam Siedlecki $^{a} \quad$ Bhumi Kinariwala $^{b}$ Sandra Sieminskic \\ aDepartment of Ophthalmology, University at Buffalo, Buffalo, NY, USA; bBrinton Vision, \\ St. Louis, MO, USA; 'Ross Eye Institute, University at Buffalo, Buffalo, NY, USA
}

\section{Keywords}

Glaucoma - Uveitis-glaucoma-hyphema syndrome - Minimally invasive glaucoma surgery · Implant removal $\cdot$ Recurrent hyphema $\cdot$ iStent

\begin{abstract}
Minimally invasive glaucoma surgery (MIGS) is a subset of glaucoma surgery that is known for its low rate of complications both intraoperatively and postoperatively compared to traditional filtering surgery. We present a case of a patient who, after receiving uncomplicated cataract extraction with posterior chamber intraocular lens implantation and concurrent iStent trabecular bypass stent implantation, experienced recurrent episodes of spontaneous microhyphema and inflammation in the late postoperative period. The patient, who had a history of mild asymmetric exfoliative glaucoma but no identifiable sources of trauma, underwent extensive examination to investigate causes of his episodic anterior segment bleeding and mild inflammation with concurrent elevated intraocular pressure. Given the circumstances of the patient's recurrent symptoms and a negative workup for conventional causes of recurrent hyphema, it was suspected that the patient's iStent was interacting with the patient's peripheral iris, causing microtrauma and hyphema. We hypothesized that this contact was potentially exacerbated by pre-existing exfoliation syndrome, which caused zonular weakness and subsequent anterior movement of the lens-iris diaphragm. After the patient's third recurrence of microhyphema and inflammation, the patient underwent removal of iStent and concurrent OMNI canaloplasty for glaucoma. Six months post-iStent explantation, the patient has had complete resolution of symptoms without recurrence of hyphema. In this case report, we outline the course of this patient's symptoms and diagnosis, discuss the potential of MIGS devices such as iStent to cause recurrent hyphema, and explore treatment options following removal.
\end{abstract}




\section{Case Reports in Ophthalmology}

\section{Introduction}

Uveitis-Glaucoma-Hyphema syndrome is a rare phenomenon caused by mechanical trauma of intraocular implants resulting in chaffing of the iris, iridocorneal angle, and/or the ciliary body. Consequently, direct damage to the uveal tissue and degradation of the blood-aqueous barrier results in episodic or chronic uveitis, pigment dispersion, iris transillumination defects (TIDs), hyphema, and microhyphemas, and is often associated with acute rise in intraocular pressure (IOP). UGH syndrome is most commonly associated with anterior chamber (AC) or iris-fixated intraocular lenses [1-3]; however, it has recently been reported in patients with posterior chamber intraocular lens (PCIOL) implants, ExPress shunts, and endocapsular tension rings [4-6].

Minimally invasive glaucoma surgery (MIGS) is a category of glaucoma surgery that was developed to minimize the complications often seen in traditional filtering. Some MIGS surgery involves implanting stents into Schlemm's canal or the suprachoroidal space to increase aqueous outflow. The first-generation iStent (Glaukos Corporation, Laguna Hills, CA, USA) is a MIGS device that bypasses the trabecular meshwork and is inserted into Schlemm's canal using an ab interno approach. The iStent is often implanted at the time of cataract surgery. To date, there have been 2 reports of recurrent hyphema occuring after iStent implantation; the first was presumed to be due to blood reflux through Schlemms canal that resolved without intervention [7], and the other was presumed to be due to iStent malposition that was resolved by surgical removal [8]. We present a unique case of a patient who experienced episodic, recurrent hyphema and uveitis with high IOP after iStent implantation despite an appropriately placed stent, verified by gonioscopy, anterior segment OCT and ultrasound biomicroscopy (UBM). Removal of the stent with concurrent OMNI canaloplasty (Sight Sciences, Menlo Park, CA, USA) resolved his symptoms.

\section{Case Presentation}

A 61-year-old Greek-American male was referred for glaucoma evaluation. He had no other ocular history and no family history of glaucoma. His past medical history included migraine and hyperlipidemia. On exam, best-corrected visual acuity (BCVA) was 20/20-2 in the right eye (OD) and 20/25+2 in the left eye (OS), pachymetry was 606 OD and 636 OS, and IOP by applanation was $18 \mathrm{~mm} \mathrm{Hg} \mathrm{OD} \mathrm{and} 28 \mathrm{~mm} \mathrm{Hg} \mathrm{OS} \mathrm{on} \mathrm{no} \mathrm{drops.} \mathrm{Anterior} \mathrm{segment} \mathrm{exam}$ showed trace pigment granules on the corneal endothelium OS only, trace cataracts in both eyes (OU), prominent exfoliative material on the pupillary margin OS without iris TIDs or phacodonesis OU. Goniosocopy showed Shaffer grade 3 angle OD with normal pigmentation and no abnormalities, and Shaffer grade 2 angle OS with heavy pigmentation of the meshwork and a Sampaolesi's line with no evidence of peripheral anterior synechiae (PAS) or neovascularization. Humphrey Visual Fields 24-2 OU was full and optical coherence tomography (OCT) of the peripapillary retinal nerve fiber layer showed no thinning OD and borderline thinning OS. He was started on a prostaglandin analog OU on presentation.

He underwent selective laser trabeculoplasty in the left eye on 2 occasions over the next 2 years due to progression of retinal nerve fiber layer thinning on OCT. Two years later, routine gonioscopy revealed narrowing of the iridocorneal angle OU, presumably from the maturing cataract, and no evidence of phacodonesis on exam. The decision was made to perform combined cataract extraction with PCIOL implantation with a first-generation iStent implantation. The surgery was performed from a superior approach by a left-handed glaucoma surgeon (SS). The iStent OS was placed inferonasally without complication, and there was no significant zonular dehiscence noted in either eye intraoperatively. The immediate postoperative

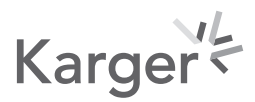




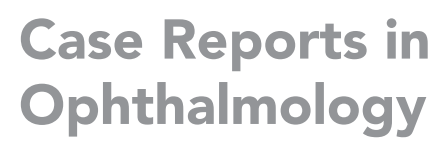

Case Rep Ophthalmol 2022;13:82-88

\begin{tabular}{l|l}
\hline DOI: $10.1159 / 000519660$ & (c) 2022 The Author(s). Published by S. Karger AG, Basel
\end{tabular}

www.karger.com/cop

Siedlecki et al.: UGH Syndrome Following iStent Implantation

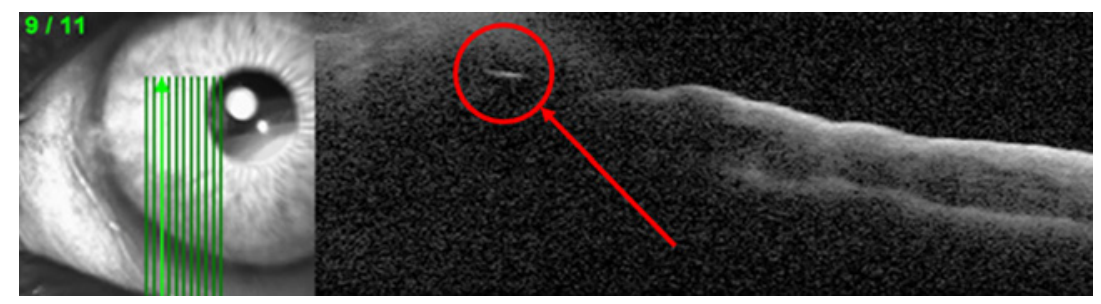

Fig. 1. Anterior segment OCT of the patient shows the iStent (circled in red) is in the appropriate position.

course was uneventful with an average left eye IOP of $20 \mathrm{~mm} \mathrm{Hg}$ at 1 month on no glaucoma medications and 17 at 3 months.

Six months postoperatively, the patient presented for an emergency visit complaining of photopsias and foggy vision around lights OS upon waking. He denied ocular trauma. BCVA was 20/20 OS, IOP was 17 OS, and 1+ punctate epithelial erosions were noted on the cornea OS. No AC cell was observed. He was given artificial tears, and his symptoms resolved.

Eightmonths postoperatively, the patient again presented to clinic complaining of photopsia and halos around lights and hazy vision OS. He noted the symptoms upon waking and denied trauma or pain. BCVA was 20/20 OD and 20/20-1 OS, IOP was $17 \mathrm{~mm} \mathrm{Hg} \mathrm{OD}$, and $19 \mathrm{~mm} \mathrm{Hg}$ OS on no drops, and the AC was deep with 1+ mixed cell and flare. The PCIOL and iStent were noted to be in good position, and there was no evidence of iris TIDs or chafing from the PCIOL. The patient was dilated and no vitreous hemorrhage or posterior cell was observed. Gonioscopy showed Schaeffer grade 4 flat iris with heavy pigmentation OS, no PAS or neovascularization of the angle, and the iStent in good position with small layered new hemorrhage (heme) around the ostium. There were no PAS around either iStent. With no cause identified for inflammation and bleeding, the patient was started on a slow taper of prednisolone acetate $1 \%$. The microhyphema resolved, but the patient was kept on prednisolone for several weeks due to persistent trace cell. Because there was no documented AC cell in the patient's visit 6 months postoperatively, this was considered the first episode of postoperative uveitis and hyphema.

Eleven months postoperatively, the patient returned for an emergency visit with a repeat of halos and haze OS only. He again reported the symptoms upon waking and denied trauma. BCVA was 20/20-1 OD and 20/20 OS, IOP was 12 OD and 22 OS, and AC showed trace cell without flare and fine pigment on corneal end othelium. Gonioscopy OS showed again a grade 4 angle, the iStent in good position on gonioscopic examination, and a small clot of heme near the iStent ostium without frank hyphema. The patient was started on brimonidine $2 \%$-timolol $0.5 \%$ twice daily (bid) OS and his prednisolone was tapered. Due to the persistent appearance of hemorrhage surrounding the iStent over 1 month postoperatively and the absence of other explanatory pathology, there was discussion that removal of the implant may be warranted if signs and symptoms did not abate. The microhyphema and inflammation resolved completely with a short course of prednisolone acetate.

Seventeen months postoperatively, the patient returned for another emergency visit with similar complaints of waking with blurry vision OS. BCVA was 20/40-1 OS and IOP was 23 on brimonidine $2 \%$-timolol $0.5 \%$ bid OS. Examination of the anterior segment revealed a quiet conjunctiva, deep AC with 2+ mainly pigmented cell, no iris TIDs, clear well-centered PCIOL in the bag, and quiet vitreous. Gonioscopy revealed Shaffer grade 4 iridocorneal angles and the iStent in good position with heme layering on the iris around the iStent inferonasally. Anterior segment OCT OS showed appropriate stent placement (Fig. 1). UBM showed an intraocular lens in good position, without evidence of in-the-bag PCIOL chafing of the iris. Since all episodes occurred presumably overnight, it was suspected that the iris was contacting the iStent upon dilation when the patient's eyes were closed. The patient was started on a trial

\section{Karger'}




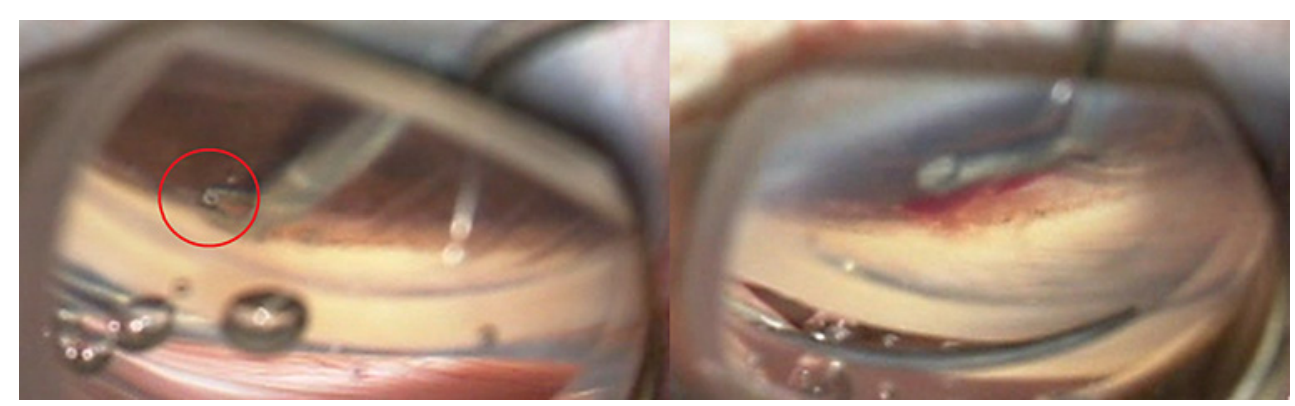

Fig. 2. Screenshots from video taken of the patient's iStent removal (circled in red in left picture) and OMNI surgery. Removal of iStent with retinal microforceps (left) and subsequent reflux of heme into AC (right). $\mathrm{AC}$, anterior chamber.

of pilocarpine $1 \%$ qhs OS to draw the iris away from the iStent. The patient inquired if his sleeping position could be exacerbating symptoms; he recalled that each episode followed sleeping on his right side, opposite the side of the affected eye. At follow-up 5 days after, the patient's vision and symptoms had improved. BCVA was 20/20-1, IOP was 14 OS on brimonidine $2 \%$-timolol $0.5 \%$ bid OS, pilocarpine $1 \%$ qhs, and prednisolone acetate $1 \% 4$ times daily (qid) OS. Slit-lamp examination showed quiet eye findings. However, the patient found pilocarpine therapy to be intolerable and requested removal of the stent. Since the patient had prior progression of glaucoma OS seen on OCT despite multiple past SLT treatments, combined surgical removal of the iStent with MIGS was discussed.

Surgical removal of the iStent with $180^{\circ}$ OMNI canaloplasty was performed 18 months following initial iStent implantation. The iStent was removed atraumatically with retinal microforceps and $180^{\circ}$ nasal OMNI canaloplasty was performed without complication. Of note, upon removal of the iStent, there was a small amount of PAS surrounding the ostium; therefore, a minimal amount of iris manipulation was required in order to free the implant from the PAS using retinal microforceps. A small defect in Schelmms canal remained in the area where the iStent was explanted (Fig. 2).

On postoperative day one, the patient's IOP OS was 14 on prednisolone acetate $1 \%$ and antibiotic drops. The AC showed trace white cell without hyphema. One week postoperatively, the patient's IOP OS was 16 on prednisolone acetate $1 \%$ with no evidence of hyphema. One month postoperatively, IOP OS was 20 on a tapering dose of prednisolone acetate $1 \%$.

Twelve weeks post-iStent removal and canaloplasty OS, the patient presented to the clinic reporting left eye heaviness. BCVA was 20/20-2 OS and IOP was 21 on no eyedrops. Slit-lamp examination showed trace white cell in the AC, no flare, and no iris TIDs. Gonioscopy showed Shaffer grade 4 angle OS with 1+ pigment and a strand of coagulated heme in the nasal angle at the site of iStent removal; this was attributed to reflux of blood through the previously mentioned Schelmm's canal defect. UBM was repeated at this visit and again showed that the PCIOL was in place in the capsular bag, without any contact between the PCIOL and the iris (Fig. 3). The patient was started on prednisolone qid OS and brimonidine $2 \%$-timolol $0.5 \%$ bid OS. The patient followed up in clinic 2 weeks later and his symptoms resolved. His IOP was $14 \mathrm{~mm} \mathrm{Hg}$ on brimonidine $2 \%$-timolol $0.5 \%$ bid OS and prednisolone qid OS. Slit-lamp examination showed a quiet eye, and gonioscopy showed no heme in the angle; that area of heme in the nasal angle had resolved. The prednisolone was tapered and the brimonidine $2 \%$-timolol $0.5 \%$ was discontinued. At his final follow-up 10 months postiStent removal and canaloplasty, he had not had a recurrence of hyphema, and his IOP remains $15 \mathrm{~mm} \mathrm{Hg}$ off of all medications.

\section{Karger's}




\section{Case Reports in Ophthalmology}

Fig. 3. Appropriate in-the-bag positioning of intraocular lens (indicated by arrow) on UBM. UBM, ultrasound biomicroscopy.

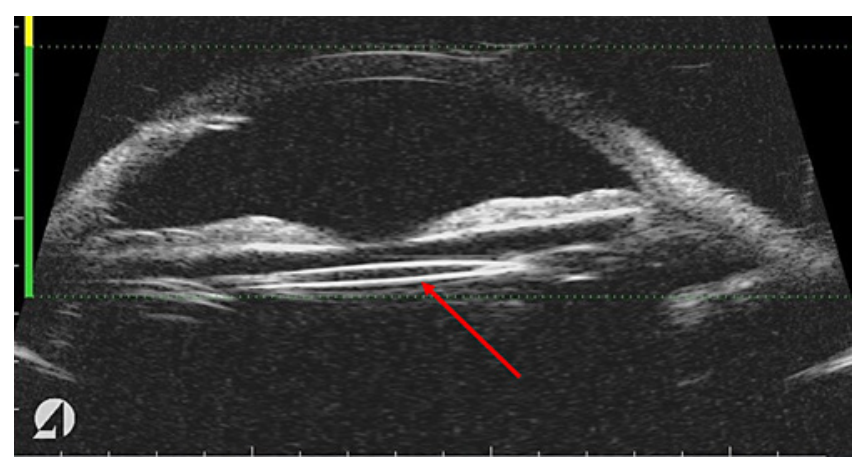

\section{Discussion}

The differential diagnosis of recurrent hyphema with concurrent rise in IOP includes recurrent trauma, stent malposition, iris rubeosis, vasculopathy, Swan syndrome (neovascularization of a scleral tunnel wound), Behcet's disease, coagulopathy or anticoagulation, and iris varices [1]. The patient's medical history and examination findings did not support these diagnoses. He did not have neovascularization of the iris, the patient denied eye rubbing, a clear corneal incision was used for his initial surgery. The recurrent nature of the patient's hyphema following insertion of surgical implant is reminiscent of UGH syndrome. The patient's UBM, which showed no contact between the PCIOL and iris, was not consistent with UGH syndrome caused by his PCIOL; however, the location of the patient's hyphema around his iStent raised suspicion for the stent as the offending agent triggering an UGH-like picture.

The iStent belongs to a growing class of MIGS devices, which offer the advantage of minimal intraoperative or postoperative complications compared to more invasive options such as trabeculectomy. Intraoperatively, the device may cause blood reflux from Schlemm's canal through the trabecular meshwork. The side effect profile is minimal and includes iStent malposition, stent obstruction, and transient hyphema $[9,10]$. Tan and $\mathrm{Au}[11]$ reported transient hyphema in $2.4 \%$ of patients $(n=42)$, Pillnaut et al. [9] reported hyphema in $2.3 \%$ of eyes $(n=43)$, and Patel et al. [12] reported hyphema in $2.3 \%$ of eyes $(n=43)$, and Vold et al. [13] reported transient hyphema in $1.9 \%$ of cases $(n=53)$. It should be noted that in the trials that cited hyphema as a side effect, all cases resolved within 1 week and with no recurrences.

There have been only two other reported cases of recurrent hyphema after iStent surgery in the current literature and only one in a patient with pseudoexfoliation syndrome. Sandhu et al. [7] reported the case of a 77-year-old woman with bilateral exfoliative glaucoma who developed recurrent microhyphema and rises in IOP upon waking from sleep 13 months and 19 months postoperatively. Khouri et al. [8] reported a case of recurrent hyphema and refractory increased IOP in an iStent patient secondary to stent malposition; surgical removal of the stent resolved the recurrent hyphema and elevated IOP.

Regarding the mechanism of recurrent hyphema in our patient and prior case reports, Sandhu et al. [7] suggested interplay between postural effects on episcleral venous pressure (EVP) relative to IOP. They proposed that when a patient lays supine, EVP increases and may exceed the IOP in the anterior chamber, causing retrograde blood flow into the AC [7]. However, the patient whom Sandhu et al. [7] described developed symptoms after sleeping on the affected side, whereas our patient developed symptoms after sleeping on the opposite, unaffected side. We did not investigate further whether there was a cause for elevated EVP with ancillary tests, such as CT or MRI of the brain, or more extensive ophthalmic diagnositcs; therefore, elevated EVP cannot be completely ruled out. We deduced that potential zonular

\section{Karger's}


dehiscence secondary to exfoliation syndrome combined with gravity when sleeping on the unaffected side caused anterior and inferonasal displacement of the iris-lens diaphragm. This positioning may have exacerbated the contact between our patient's iris and iStent, leading to the focal layered hyphema we observed near the stent ostium inferonasally. This mechanical interaction between uveal tissue and implant resembles microtrauma caused by other ocular implants in an UGH picture.

Our patient's finding of heme in the angle 3 months postoperatively after OMNI canaloplasty and removal of iStent was thought to be reflux of blood through a defect in Schlemm's canal and angle structures following removal of the iStent. OMNI is a relatively new device, and few studies have been published on it and its adverse effects profile; however, Tracer et al. [14] did note hyphema as an adverse effect within 1 month of OMNI canaloplasty in $1.7 \%$ of their patient group. Further studies should be done on the safety profile and longer term adverse effects of OMNI canaloplasty.

In conclusion, we present a case of UGH syndrome in a patient with exfoliative glaucoma after uncomplicated cataract extraction, intraocular lens, and iStent placement. Removal of the iStent led to a resolution of the UGH syndrome, and concurrent OMNI canaloplasty resulted in adequate IOP control off of medications. We hypothesized that the iStent caused localized trauma to the patient's iris due to a combination of mechanical stress potentiated by exfoliation syndrome combined with nocturnal positional changes. That is, subclinical zonular weakness combined with positional changes overnight stimulated friction and shearing by the iStent on the surrounding tissue. Resolution of symptoms by 6 months after iStent explantation without recurrence further suggests that the iStent was the causative agent seen in this patient. It is the purpose of this case report to illustrate that iStent, like other ocular implants, may cause symptoms reminiscent of an UGH-like syndrome. In addition, this is the second of case reports involving recurrent hyphema with iStent in exfoliative glaucoma. This may highlight the fact that exfoliative glaucoma could lead to increased lens-iris diaphragm movement, especially at night, which could lead to issues with iStent and other MIGS.

\section{Acknowledgments}

There are no nonauthor contributors to acknowledge.

\section{Statement of Ethics}

The patient in this study has given his written informed consent to publish this case, including history elements and images of his surgery. Ethics approval by an Institutional Review Board or other committee is not required for case reports.

\section{Conflict of Interest Statement}

The authors have no conflicts of interest to declare.

\section{Funding Sources}

This study received no funding from any outside sources.

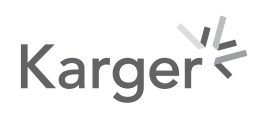




\section{Author Contributions}

Adam Siedlecki contributed to the chart review, literature search, wrote and edited the manuscript draft, and edited the resubmission. Bhumi Kinariwala contributed to writing and editing the manuscript draft and saw the patient in the clinic. Sandra Sieminski saw the patient in clinic, performed the implantation and explantation surgeries, wrote and edited the manuscript, and edited the resubmission.

\section{Data Availability Statement}

All relevant data generated and analyzed during this study are included in this article and online supplementary material (for all online suppl. material, see www. karger.com/doi/10.1159/000519660). Further inquiries can be directed to the corresponding author.

\section{References}

1 Zemba M, Camburu G. Uveitis-glaucoma-hyphaema syndrome. General review. Rom J Ophthalmol. 2017;61(1): $11-7$.

2 Zhang L, Hood CT, Vrabec JP, Cullen AL, Parrish EA, Moroi SE. Mechanisms for in-the-bag uveitis-glaucomahyphema syndrome. J Cataract Refract Surg. 2014;40(3):490-2.

3 Shweikh Y, Ameen S, Mearza A. Complications secondary to cosmetic artificial iris anterior chamber implants: a case report. BMC Ophthalmol. 2015;15:97.

4 Hou A, Hasbrook M, Crandall D. A case of uveitis-hyphema-glaucoma syndrome due to EX-PRESS glaucoma filtration device implantation. J Glaucoma. 2019;28(10):e159-61.

5 Cheung AY, Price JM, Heidemann DG, Hart JC Jr. Uveitis-glaucoma-hyphema syndrome caused by dislocated Cionni endocapsular tension ring. Can J Ophthalmol. 2018;53(5):e213-4.

6 Badakere SV, Senthil S, Turaga K, Garg P. Uveitis-glaucoma-hyphaema syndrome with in-the-bag placement of intraocular lens. BMJ Case Rep. 2016;2016:bcr2015213745.

7 Sandhu S, Arora S, Edwards MC. A case of delayed-onset recurrent hyphema after iStent surgery. Can J Ophthalmol. 2016 Dec;51(6):e165-7.

8 Khouri AS, Megalla MM. Recurrent hyphema following iStent surgery managed by surgical removal. Can J Ophthalmol. 2016 Dec;51(6):e163-5.

9 Pillnaut L, Erb C, Jünemann A, Kimmich F. Micro-invasive glaucoma surgery (MIGS): a review of surgical procedures using stents. Clin Ophthalmol. 2017;11:1583-600.

10 Chen DZ, Sng CCA. Safety and efficacy of microinvasive glaucoma surgery. J Ophthalmol. 2017;2017:3182935.

11 Tan SZ, Au L. Manchester iStent study: 3-year results and cost analysis. Eye. 2016;30(10):1365-70.

12 Patel I, de Klerk TA, Au L. Manchester iStent study: early results from a prospective UK case series. Clin Exp Ophthalmol. 2013 Sep-0ct;41(7):648-52.

13 Vold SD, Voskanyan L, Tetz M, Auffarth G, Masood I, Au L, et al. Newly diagnosed primary open-angle glaucoma randomized to 2 trabecular bypass stents or prostaglandin: outcomes through 36 months. Ophthalmol Ther. 2016;5(2):161-172.

14 Tracer N, Dickerson JE, Radcliffe NM. Circumferential viscodilation ab interno combined with phacoemulsification for treatment of open-angle glaucoma: 12-month outcomes. Clin Ophthalmol. 2020;14:1357-1364.

\section{Karger'}

